\title{
Article \\ COVID-19 Lockdown in Belgrade: Impact on Air Pollution and Evaluation of a Neural Network Model for the Correction of Low-Cost Sensors' Measurements
}

\author{
Ivan Vajs ${ }^{1,2, *}$, Dejan Drajic ${ }^{1,2,3}$ and Zoran Cica ${ }^{1}$ (D) \\ 1 School of Electrical Engineering, University of Belgrade, Bulevar Kralja Aleksandra 73, 11120 Belgrade, Serbia; \\ ddrajic@etf.bg.ac.rs (D.D.); zoran.cica@etf.bg.ac.rs (Z.C.) \\ 2 Innovation Center, School of Electrical Engineering, University of Belgrade, Bulevar Kralja Aleksandra 73, \\ 11120 Belgrade, Serbia \\ 3 DunavNET, DNET Labs, Bulevar Oslobodjenja 133, 21000 Novi Sad, Serbia \\ * Correspondence: ivan.vajs@ic.etf.bg.ac.rs; Tel.: +381-11-3218-455
}

Citation: Vajs, I.; Drajic, D.; Cica, Z. COVID-19 Lockdown in Belgrade: Impact on Air Pollution and Evaluation of a Neural Network Model for the Correction of Low-Cost Sensors' Measurements. Appl. Sci. 2021, 11, 10563. https://doi.org/ 10.3390/app112210563

Academic Editor: Kyung Hwan Kim

Received: 28 September 2021

Accepted: 8 November 2021

Published: 10 November 2021

Publisher's Note: MDPI stays neutral with regard to jurisdictional claims in published maps and institutional affiliations.

Copyright: () 2021 by the authors. Licensee MDPI, Basel, Switzerland. This article is an open access article distributed under the terms and conditions of the Creative Commons Attribution (CC BY) license (https:// creativecommons.org/licenses/by/ $4.0 /)$.

\begin{abstract}
In this paper, we explore the impact of the COVID-19 lockdown in Serbia on the air pollution levels of $\mathrm{CO}, \mathrm{NO}_{2}$ and $\mathrm{PM}_{10}$ alongside the possibility for low-cost sensor usage during this period. In the study, a device with low-cost sensors collocated with a reference public monitoring station in the city of Belgrade is used for the same period of 52 days in 2019 (pre-COVID-19 period), 2020 (COVID-19 lockdown) and 2021 (post-COVID-19 period). Low-cost sensors' measurements are improved by using a convolutional neural network that applies corrections of the influence of temperature and relative humidity on the low-cost sensors. As a result of this study we have noticed a remarkable decrease in $\mathrm{NO}_{2}$ (primarily related to traffic density), while on the other hand $\mathrm{CO}$ and $\mathrm{PM}_{10}$, related to domestic heating sources and heating plants, showed constant or slightly higher levels. The obtained results are in accordance with other published work in this area. The low-cost sensors have shown a satisfactory correlation with the reference $\mathrm{CO}$ measurements during the lockdown, while the $\mathrm{NO}_{2}$ and $\mathrm{PM}_{10}$ measurements of 2020 were corrected using a convolutional neural network trained on meteorological and pollutant data from 2019. The results include an improvement of 0.35 for the $\mathrm{R} 2$ of $\mathrm{NO}_{2}$ and an improvement of 0.13 for the $\mathrm{R} 2$ of $\mathrm{PM}_{10}$, proving that our neural network model trained on data from 2019 can improve the performance of the sensor in the lockdown period in 2020. This means that our neural network model is very robust, as it exhibits good performance even in the case where training data from the prior year (2019) are used in the following year (2020) in very different environment circumstances-a lockdown.
\end{abstract}

Keywords: air pollution monitoring; COVID-19; emergency lockdown; low-cost PM and gas sensors $\left(\mathrm{CO}\right.$ and $\left.\mathrm{NO}_{2}\right)$; neural network; sensor calibration

\section{Introduction}

Due to the COVID-19 pandemic, in order to protect citizens and stop the virus spreading, most governments around the world declared a state of emergency and conducted a partial or total lockdown for a certain period. In Serbia, an emergency lockdown was introduced on 16 March 2020 and lasted until 6 May 2020 (i.e., 52 days) [1]. During that period, some days had tighter emergency measures (complete lockdown) while during other days there was a partial lockdown. Generally, Belgrade is a moderately polluted city, mostly affected by traffic and transport, construction, industrial activities, dust and domestic-heating-related pollution. As a consequence of the lockdown, vehicle traffic volume, manufacturing, construction and industrial activities were reduced. To understand the influence of these factors on pollution, it is of interest to analyze air quality during the emergency lockdown and compare it with the preceding and following periods.

There are many analyses, studies and pieces of research related to the influence of COVID-19 lockdowns on air quality [2]. In [3], the authors compare the consecutive 
intervals that occur immediately pre-COVID, COVID and post-COVID in terms of their influence on air quality in the city of Novi Sad (Serbia) for $\mathrm{PM}_{2.5}, \mathrm{PM}_{10}, \mathrm{CO}$ and $\mathrm{NO}_{\mathrm{x}}$. The authors have noticed a significant reduction in $\mathrm{NO}_{2}$, while $\mathrm{CO}$ and $\mathrm{PM}_{10}$ mostly remained on the same level (depending on the location in Novi Sad). $\mathrm{PM}_{2.5}, \mathrm{PM}_{10}, \mathrm{NO}_{2}$ and $\mathrm{O}_{3}$ concentrations are explored and compared for the same periods in 2019 and 2020 (during the COVID lockdown in Florence, Pisa and Lucca in Italy) in [4]. In this study, a significant reduction in $\mathrm{NO}_{2}$ was observed, while the pollution level of $\mathrm{O}_{3}$ remained the same; $\mathrm{PM}_{10}$, in most of the analyzed cases, remained on the same level. Major urban cities are explored in [5], and a reduction in $\mathrm{NO}_{2}$ pollution is highly noticeable (in comparison to 2019) in the cities where there was a strict lockdown (Madrid, Milan and Paris), while in the cities with a partial lockdown this decrease was much lower (Warsaw, Bismarck and Lincoln). The French National Institute (INERIS) has conducted a comprehensive study related to $\mathrm{NO}_{2}$ and $\mathrm{PM}_{10}$ around Europe, and a decrease in $\mathrm{NO}_{2}$ pollution was also noticed, but the $\mathrm{PM}_{10}$ pollution was shown to depend on the observed region (an increase/decrease was observed) [6]. The same behavior, regarding $\mathrm{NO}_{2}$ and $\mathrm{PM}$ pollution levels, is also noticed in Canada and presented in [7]. The impact of the COVID-19 lockdown on traffic-related air pollution ( $\mathrm{PM}_{2.5}$, black carbon (BC), NO, $\mathrm{NO}_{2}$ and $\left.\mathrm{CO}\right)$ in a northwestern US city is evaluated in [8]. Based on the multiple studies in this area, it was concluded that road traffic is a major urban pollutant source. The measurements were conducted near a major freeway. During the lockdown period, the median traffic volume decreased by a third. This caused a decrease in the level of pollutants, where the median $\mathrm{BC}$ concentration decreased by a fourth, $\mathrm{PM}_{2.5}, \mathrm{NO}$ and $\mathrm{NO}_{2}$ decreased by a third and $\mathrm{CO}$ decreased by a sixth in comparison to the pre-COVID-19 state.

The modeling of the influence of weather conditions on low-cost air quality sensors is quite present in modern research. More specifically, artificial neural networks (ANNs), as very promising machine learning algorithms, can be found in several papers related to low-cost sensors. In [9], the authors use a deep ANN consisting of convolutional, fully connected and long short-term memory layers, and train it on the $\mathrm{O}_{3}, \mathrm{PM}_{2.5}$ and $\mathrm{PM}_{10}$ measurements, taking into consideration various weather conditions. They have concluded that by training a network using reference, low-cost measurements, such as wind speed, wind direction, temperature and relative humidity, the error of low-cost sensors can be drastically decreased. The AirNet model was developed in [10] and uses dual encoder neural networks to calibrate $\mathrm{CO}$ and $\mathrm{O}_{3}$ low-cost sensors. The developed AirNet architecture has been proven to be superior via comparison to several baseline algorithms. A mobile $\mathrm{PM}_{2.5}$ sensing system was developed and calibrated using fully connected ANNs in [11]. It considers relative humidity and temperature in addition to $\mathrm{PM}_{2.5}$ measurements and was shown to greatly increase the accuracy of the developed system. In [12], the authors explore three different machine learning algorithms for the calibration of low-cost $\mathrm{CO}$ and $\mathrm{O}_{3}$ sensors based on a number of pollutant measurements and weather conditions. Univariate and multivariate linear regression are implemented as well as fully connected ANNs. Several training algorithms for the ANNs are implemented as well, and it was concluded that the ANN outperforms the linear algorithms for both pollutants. The authors have explored the possibility of applying linear models and fully connected ANNs to data collected from a location different than the one used for the acquisition of training data in [13]. $\mathrm{O}_{3}$ and $\mathrm{CO}_{2}$ measurements have been observed. It was shown that the ANN is overall more effective, but that there are some exceptions influenced by the selection of training and test scenarios. An evaluation of linear models and fully connected ANNs is performed in [14], with an assortment of observed pollutants: $\mathrm{O}_{3}, \mathrm{CO}$, $\mathrm{CO}_{2}$ and $\mathrm{CH}_{4}$. It was shown that the $\mathrm{ANN}$ has better performance when the modeling of complex nonlinear behavior is concerned, using a wide range of pollutant and weather condition measurements. Regardless of the variety of the papers that have covered air quality sensor calibration, to the best of the authors' knowledge no paper has attempted to train algorithms on data from a single year and evaluate it on data that is gathered a year later during a different day-to-day regime. 
The scope and contributions of our research are twofold. The first part is to explore the impact of the COVID-19 lockdown in Serbia on air pollution levels. The second part includes a device with low-cost sensors which is collocated with a reference public monitoring station. The goal here is to validate the device measurements by comparing them with reference measurements, and to apply corrections due to the influence of temperature and relative humidity on the measurements by using convolutional ANNs. Our results show that by using data from the year preceding the COVID-19 lockdown, a convolutional ANN model can be trained and successfully applied to low-cost sensors' measurements during the lockdown period. This shows that our model can be applied to low-cost measurements that are acquired a year later than the ones used for training data. This indicates that the sensors can be successfully used for multiple years and that our calibration algorithm can be implemented at least one year after it is trained (showing a decent level of robustness). Furthermore, the pollutant concentrations are different in 2019 and 2020 (COVID-19 lockdown), and the algorithm was trained in 2019 and tested in 2020, showing that it is useful even if the regimes during training and testing are different.

The rest of the paper is organized as follows: in Section 2 our research methods are presented. The results and subsequent discussion are presented in Section 3. Finally, Section 4 concludes the paper.

\section{Methods}

The IoT (Internet of Things)-based air quality monitoring ekoNET device AQ10x (DunavNET, AQ103, Novi Sad, Serbia) with low-cost sensors for outdoor air quality measurements [15] has been collocated with a reference public monitoring station in Belgrade (Serbia). The system contains the following components: an AQ10x device (Figure 1), a cloud database for permanent data storage, a data analytics engine (Microsoft Azure), a visualization engine, an administration module and a web application. The AQ10x is a portable device that can be installed both indoors and outdoors, as well as on vehicles to enable larger coverage. The device's power consumption is $2.5 \mathrm{~W}$, weight is $1.5 \mathrm{~kg}$ and dimensions are $180 \times 180 \times 265 \mathrm{~mm}^{3}$. AQ10x devices support different transmission technologies (WiFi, BLE (Bluetooth Low Energy), LoRa (Long Range), SigFox, GPRS (General Packet Radio Service), 3G, LTE (Long-Term Evolution) and NB-IoT (Narrowband Internet of Things), and measurements are sent to the back-end server via one of the selected communication modules. The average payload for one data transmission (one measurement period) is $100 \mathrm{~B}$. The transmission period can be set by the user, and for a recommended period of $1 \mathrm{~min}$ the total monthly payload is about $4.5 \mathrm{MB}$. The data are visualized in real time using the web application, which provides a rich set of tools for data processing (averaging, filtering, noise elimination, etc.). In comparison to the public monitoring station, for the same set of observed pollutants, the cost of the low-cost device is about 25 times lower (i.e., for the cost of 1 public reference station, 25 low-cost devices can be purchased). Furthermore, the public reference station additionally requires a significantly higher amount of maintenance.

The device contains the following B4 series Alphasense electrochemical gas sensors, intended to monitor air quality in urban areas [16]: CO-B4 gas sensor (measurement range: 0-50 ppm, unit ppm or $\mathrm{mg} / \mathrm{m}^{3}$, accuracy $\pm 2 \% \mathrm{FS}$ ), $\mathrm{NO}_{2}-\mathrm{B} 43 \mathrm{~F}$ gas sensor (measurement range: $0-20 \mathrm{ppm}$, unit $\mathrm{ppb}$ or $\mu \mathrm{g} / \mathrm{m}^{3}$, accuracy $\pm 2 \% \mathrm{FS}$ ), air temperature (t) and relative humidity (RH) sensors (Bosch BME 280) in addition to $\mathrm{PM}_{1}, \mathrm{PM}_{2.5}$ and $\mathrm{PM}_{10}$ Plantower PMS7003 optical counters (measurement range: $0 \sim 1000 \mu \mathrm{g} / \mathrm{m}^{3}$, unit $\mu \mathrm{g} / \mathrm{m}^{3}$, accuracy $\pm 2 \%$ FS) [17].

The measurement point is located in the biggest municipality in Belgrade (and in Serbia), i.e., New Belgrade. The station is located a few tens of meters away from one of the main intersections in New Belgrade with a high traffic volume, and a few hundred meters from the Sava River as well as the Ada Lake. The main sources of air pollution in this area are transport (traffic), a heating plant (about $1 \mathrm{~km}$ from the measurement point), industrial and construction activities, domestic heating and dust. The device sampling resolution 
is 1 minute, and data are sent via a GPRS network to the back-end server in the cloud, where data are stored and processed. Obtained values are averaged for each hour and statistically correlated to values captured from the public monitoring station in the same time intervals. Results are collected and analyzed for the periods of 16 March-6 May 2019, 16 March-6 May 2020 (COVID lockdown) and 16 March-6 May 2021. In this study, CO, $\mathrm{NO}_{2}$ and $\mathrm{PM}_{10}$ measurements are used, alongside weather data. The data from the period of the Aralkum desert storm are excluded from the 2020 dataset to avoid interference with the results [3].

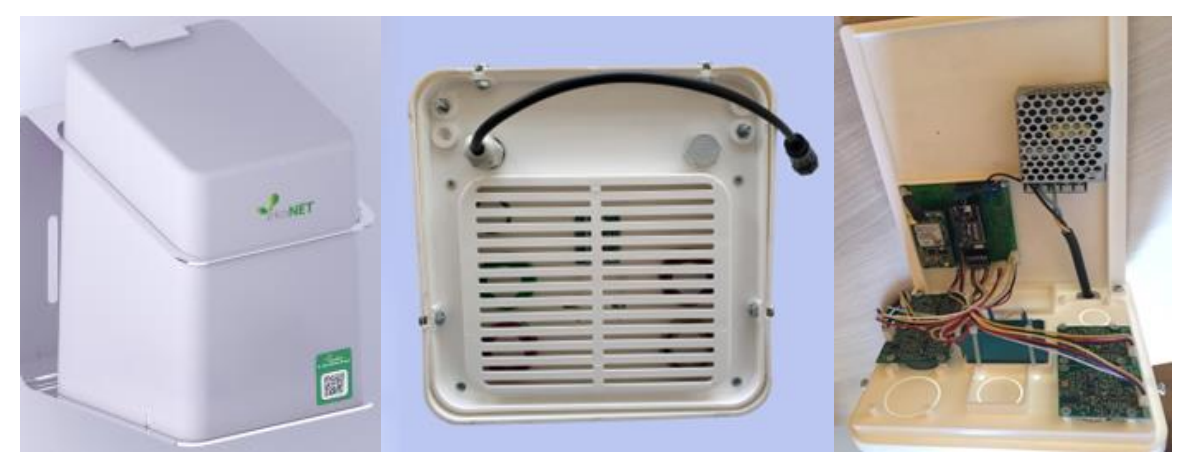

Figure 1. Air quality ekoNET device, AQ10x. The whole device is shown on the left, with the bottom view of the device shown in the middle and the interior shown on the right. The sensors are placed at the bottom of the device, with access to open air, while the power supply and the microprocessor with the data transfer module are placed on the back.

The raw measurement data are collected from low-cost sensors. Since low-cost sensors do not provide highly precise measurements, the collected measurements should be corrected. As we stated in the Introduction section, we used convolutional ANNs for this purpose. The goal regarding the ANN calibration performed in this paper is to train the network to correct the raw low-cost sensors' measurements, using air temperature and relative humidity measurements. To evaluate the model in a realistic scenario, the model is trained on data from 2019 and tested on data from 2020 and 2021. The model is also trained on data from both 2019 and 2020 and tested on the 2021 measurements. In this manner, we test whether the calibration done in one year could be applied in the following year, and if the calibration done during regular activities (2019) could be applied when there was a lockdown (2020).

The gathered raw pollutant measurements from each of the three years are initially corrected using the Environmental Protection Agency (EPA) co-location method, with linear regression (LR) between the raw and reference values obtained in the two-week period preceding the observed one (LR performed on the data gathered from 2 March to 16 March of the respective year) [18]. After this, each measured variable is grouped into sequences with a length of 24 . A window of $24 \mathrm{~h}$ ( 1 day) is moved through the data of length 1248 for a single year (number of hours in the observed main interval), with a stride of 1 hour creating a $1224 \times 24$ matrix of data. The corrected sensor measurements, $\mathrm{RH}$ and $\mathrm{t}$ data are concatenated, resulting in a $1224 \times 24 \times 3$ matrix of input data created for each year. The output data for each year are $1224 \times 24$ matrices containing reference measurements. The data instances (1224 per year), each of shape $24 \times 3$, are the input to the developed convolutional ANN, and the reference measurements for the respective $24 \mathrm{~h}$ are the output of the network.

The architecture of the convolutional ANN used for the correction of the influence of relative humidity and temperature on measurements is given in Table 1. 
Table 1. Convolutional network architecture.

\begin{tabular}{ll}
\hline \multicolumn{1}{c}{ Layer Type } & \multicolumn{1}{c}{ Layer Properties } \\
\hline Conv1D & filters $=64$, kernel size $=3$, strides $=1$ \\
Conv1D & filters $=64$, kernel size $=3$, strides $=1$ \\
Batch normalization & $/$ \\
Max pooling & pool size $=2$, strides $=2$ \\
Dropout & drop rate $=0.5$ \\
Conv1D & filters $=128$, kernel size $=3$, strides $=1$ \\
Conv1D & filters $=128$, kernel size $=3$, strides $=1$ \\
Batch normalization & $/$ \\
Max pooling & pool size $=2$, strides $=2$ \\
Dropout & drop rate $=0.5$ \\
Conv1D & filters $=256$, kernel size $=3$, strides $=1$ \\
Batch normalization & $/$ \\
Max pooling & pool size $=2$, strides $=2$ \\
Dropout & drop rate $=0.5$ \\
Conv1D transpose & filters $=256$, kernel size $=3$, strides $=1$ \\
Batch normalization & $/$ \\
Upsampling & upsampling factor $=2$ \\
Dropout & drop rate $=0.5$ \\
Conv1D transpose & filters $=128$, kernel size $=3$, strides $=1$ \\
Conv1D transpose & filters $=128$, kernel size $=3$, strides $=1$ \\
Batch normalization & $/$ \\
Upsampling & upsampling factor $=2$ \\
Dropout & drop rate $=0.5$ \\
Conv1D transpose & filters $=64$, kernel size $=3$, strides $=1$ \\
Conv1D transpose & filters $=64$, kernel size $=3$, strides $=1$ \\
Batch normalization & $/$ \\
Upsampling & upsampling factor $=2$ \\
Dropout & drop rate $=0.5$ \\
Conv1D transpose & filters $=1$, kernel size $=3$, strides $=1$ \\
\hline &
\end{tabular}

This convolutional ANN is trained for each pollutant separately, but the architecture itself remains the same for all pollutants. To evaluate the algorithm, the commonly used metrics R2 and root mean square error (RMSE) are used [19].

\section{Results and Discussion}

Table 2 shows the evaluation metrics for the performed measurement corrections for $\mathrm{NO}_{2}$ and $\mathrm{PM}_{10}$. The agreement between the raw and reference $\mathrm{CO}$ measurements is initially quite high for each year ( $\mathrm{R} 2$ ranging from 0.833 to 0.894 ), and no significant improvement is achieved for this pollutant using the correction of the raw measurements described in the previous section. Because of this, results regarding the $\mathrm{CO}$ measurements are not shown in Table 2.

The presented results show that, for $\mathrm{NO}_{2}$, every train/test scenario for the ANN shows an improvement over the simple LR correction results. The most prominent improvement for $\mathrm{NO}_{2}$ is achieved when the $\mathrm{ANN}$ is trained on data from 2019 and evaluated on data from 2020. It is also notable that the results for the test year 2021 are better when data from both 2019 and 2020 are used as the training set. When it comes to the results for the $\mathrm{PM}_{10}$, the most prominent improvement is present when the ANN is trained on 2019 data and tested on 2020 data. The R2 factor for $\mathrm{PM}_{10}$ is also improved for 2021, but only when the ANN is trained on 2020 data, and there seems to be an increase in the RMSE parameter. All of these observations can be explained when taking into consideration the nature of the sensors alongside the values of the measured pollutants and weather parameters. The mean, median and standard deviation values are shown in Table 3, for each year and each measured variable. In the remainder of this section, we explain in more detail the observations made for the results shown in Table 2. 
Table 2. Evaluation metrics for different train/test scenarios.

\begin{tabular}{|c|c|c|c|c|c|c|}
\hline & \multirow{2}{*}{\multicolumn{2}{|c|}{ Evaluation Scenario }} & \multicolumn{4}{|c|}{ Pollutant } \\
\hline & & & \multicolumn{2}{|c|}{$\mathrm{NO}_{2}$} & \multicolumn{2}{|c|}{$\mathbf{P M}_{10}$} \\
\hline & Train Set & Test Set & $\mathbf{R 2}$ & $\operatorname{RMSE}\left(\mu \mathrm{g} / \mathrm{m}^{3}\right)$ & R2 & $\operatorname{RMSE}\left(\mu \mathrm{g} / \mathrm{m}^{3}\right)$ \\
\hline \multirow{3}{*}{ LR } & 2.3-16.3.2019. & 16.3-6.5.2019. & 0.728 & 11.416 & 0.615 & 15.644 \\
\hline & 2.3-16.3.2020. & 16.3-6.5.2020. & 0.260 & 14.487 & 0.699 & 14.903 \\
\hline & 2.3-16.3.2021. & 16.3-6.5.2021. & 0.601 & 13.061 & 0.299 & 16.689 \\
\hline \multirow{4}{*}{ ANN } & $16.3-6.5 .2019$. & $16.3-6.5 .2020$. & 0.606 & 9.123 & 0.741 & 13.950 \\
\hline & 16.3-6.5.2020. & 16.3-6.5.2021. & 0.614 & 11.292 & 0.438 & 23.396 \\
\hline & 16.3-6.5.2019. & 16.3-6.5.2021. & 0.652 & 10.460 & 0.157 & 18.185 \\
\hline & $\begin{array}{c}\text { 16.3-6.5. } \\
\text { 2019. \& } 2020 \text {. }\end{array}$ & 16.3-6.5.2021. & 0.671 & 10.555 & 0.255 & 19.211 \\
\hline
\end{tabular}

Table 3. Mean, median and standard deviation values for the measured variables.

\begin{tabular}{|c|c|c|c|c|c|c|}
\hline \multicolumn{7}{|c|}{$\mathrm{CO}$} \\
\hline Year & $\begin{array}{c}\text { Ref. } \\
\text { Mean } \\
\left(\mathrm{mg} / \mathrm{m}^{3}\right)\end{array}$ & $\begin{array}{c}\text { Ref. } \\
\text { Median } \\
\left(\mathrm{mg} / \mathrm{m}^{3}\right)\end{array}$ & $\begin{array}{c}\text { Ref. } \\
\text { Std } \\
\left(\mathrm{mg} / \mathrm{m}^{3}\right)\end{array}$ & $\begin{array}{c}\text { LR } \\
\text { Mean } \\
\left(\mathrm{mg} / \mathrm{m}^{3}\right)\end{array}$ & $\begin{array}{c}\text { LR } \\
\text { Median } \\
\left(\mathrm{mg} / \mathrm{m}^{3}\right)\end{array}$ & $\begin{array}{c}\text { LR } \\
\text { Std } \\
\left(\mathrm{mg} / \mathrm{m}^{3}\right)\end{array}$ \\
\hline 2019 & 0.33 & 0.28 & 0.18 & 0.35 & 0.30 & 0.17 \\
\hline 2020 & 0.64 & 0.60 & 0.13 & 0.72 & 0.67 & 0.15 \\
\hline 2021 & 0.50 & 0.43 & 0.18 & 0.54 & 0.48 & 0.18 \\
\hline \multicolumn{7}{|c|}{$\mathrm{NO}_{2}$} \\
\hline Year & $\begin{array}{c}\text { Ref. } \\
\text { Mean } \\
\left(\mu \mathrm{g} / \mathrm{m}^{3}\right)\end{array}$ & $\begin{array}{c}\text { Ref. } \\
\text { Median } \\
\left(\mu \mathrm{g} / \mathrm{m}^{3}\right)\end{array}$ & $\begin{array}{c}\text { Ref. } \\
\text { Std } \\
\left(\mu \mathrm{g} / \mathrm{m}^{3}\right)\end{array}$ & $\begin{array}{c}\text { LR } \\
\text { Mean } \\
\left(\mu \mathrm{g} / \mathrm{m}^{3}\right)\end{array}$ & $\begin{array}{c}\text { LR } \\
\text { Median } \\
\left(\mu \mathrm{g} / \mathrm{m}^{3}\right)\end{array}$ & $\begin{array}{c}\text { LR } \\
\text { Std } \\
\left(\mu \mathrm{g} / \mathrm{m}^{3}\right)\end{array}$ \\
\hline 2019 & 24.73 & 17.78 & 20.52 & 27.84 & 22.74 & 19.90 \\
\hline 2020 & 9.76 & 5.92 & 13.57 & 13.82 & 14.80 & 14.50 \\
\hline 2021 & 23.04 & 18.45 & 17.54 & 27.27 & 26.02 & 19.06 \\
\hline \multicolumn{7}{|c|}{$\mathbf{P M}_{10}$} \\
\hline Year & $\begin{array}{c}\text { Ref. } \\
\text { Mean } \\
\left(\mu \mathrm{g} / \mathrm{m}^{3}\right)\end{array}$ & $\begin{array}{c}\text { Ref. } \\
\text { Median } \\
\left(\mu \mathrm{g} / \mathrm{m}^{3}\right)\end{array}$ & $\begin{array}{c}\text { Ref. } \\
\text { Std } \\
\left(\mu \mathrm{g} / \mathrm{m}^{3}\right)\end{array}$ & $\begin{array}{c}\text { LR } \\
\text { Mean } \\
\left(\mu \mathrm{g} / \mathrm{m}^{3}\right)\end{array}$ & $\begin{array}{c}\text { LR } \\
\text { Median } \\
\left(\mu \mathrm{g} / \mathrm{m}^{3}\right)\end{array}$ & $\begin{array}{c}\text { LR } \\
\text { Std } \\
\left(\mu \mathrm{g} / \mathrm{m}^{3}\right)\end{array}$ \\
\hline 2019 & 29.98 & 26.00 & 18.45 & 32.23 & 26.00 & 18.35 \\
\hline 2020 & 29.03 & 24.40 & 19.16 & 22.25 & 19.81 & 7.99 \\
\hline 2021 & 30.27 & 25.18 & 18.46 & 36.57 & 33.64 & 9.95 \\
\hline \multicolumn{7}{|c|}{ Meteorological Data } \\
\hline Year & $\begin{array}{c}\text { RH } \\
\text { Mean } \\
(\%)\end{array}$ & $\begin{array}{c}\text { RH Median } \\
(\%)\end{array}$ & $\begin{array}{l}\text { RH } \\
\text { Std } \\
(\%)\end{array}$ & $\begin{array}{c}t \\
\text { Mean } \\
\left({ }^{\circ} \mathrm{C}\right)\end{array}$ & $\begin{array}{c}\mathbf{t} \\
\text { Median } \\
\left({ }^{\circ} \mathrm{C}\right)\end{array}$ & $\begin{array}{c}t \\
\text { Std } \\
\left({ }^{\circ} \mathrm{C}\right)\end{array}$ \\
\hline 2019 & 58.78 & 57.00 & 21.20 & 13.33 & 12.70 & 5.01 \\
\hline 2020 & 52.78 & 49.00 & 19.60 & 12.29 & 12.60 & 6.31 \\
\hline 2021 & 60.77 & 61.34 & 19.55 & 10.96 & 10.19 & 6.06 \\
\hline
\end{tabular}

The values given in Table 3 can explain many phenomena regarding the influence of the state of emergency, as well as provide an explanation for the results shown in Table 2.

Firstly, let us observe the measurements of $\mathrm{CO}$. The values of the mean and median values of the $\mathrm{CO}$ reference measurements are the highest in 2020. This can be explained by the larger number of people staying indoors, because of the state of emergency, in comparison to 2019 and 2021. The observed period in each year includes the heating season, which, in addition to the larger number of people staying indoors in 2020, could explain the observable increase in the $\mathrm{CO}$ measurements. 
When it comes to the $\mathrm{NO}_{2}$ measurements, quite a significant decline in the mean, median and standard deviation values can be observed in 2020 for both the reference and low-cost sensors. This is also consequence of the state of emergency, since the main source of $\mathrm{NO}_{2}$ is traffic, which was significantly reduced during the state of emergency. The low values of $\mathrm{NO}_{2}$ are also responsible for the poor correlation between the reference and LR data ( $\mathrm{R} 2$ factor of 0.260 ). The low-cost $\mathrm{NO}_{2}$ sensor tends to have trouble with low concentrations of $\mathrm{NO}_{2}$, but the implemented ANN managed to pick up on these dependencies as well as the influence of RH and t on the measurements. Furthermore, the ANN trained on 2019 data that was applied to 2020 data shows a significant improvement of the raw measurements, implying that the behavior of the $\mathrm{NO}_{2}$ sensor, although not perfect, is consistent through 2019 and 2020. The achieved improvement can be seen in Figures 2 and 3, with Figure 2 containing the results of the LR performed in 2020 and Figure 3 containing the results of the ANN trained on 2019 data and applied to 2020 data.

Figures 2 and 3 show not only a clear improvement in regard to the scattered points being closer to the $y=x$ line, but the histogram shape as well. The consistent behavior of the low-cost $\mathrm{NO}_{2}$ sensor can also be observed in 2021. The initial correlation (after only the LR correction) is a lot better than in 2020, since the traffic (and therefore $\mathrm{NO}_{2}$ concentration) was closer to the regular regime. The improvement achieved using the ANN is still present, but less prominent than in 2020. It is also important to note that a better improvement was achieved when the ANN was trained on the data from both 2019 and 2020, as opposed to the case where the training set contained only 2020 data. This is an expected result because the low-cost $\mathrm{NO}_{2}$ sensor seems to have consistent behavior throughout the years, and a larger training dataset implies a better correspondence of the algorithm with the underlying dependencies. The achieved results can be seen in the following figures, with Figure 4 showing the results of the LR performed in 2021 and Figure 5 showing the results of the ANN that was trained on 2019 and 2020 data.

Higher concentrations of the $\mathrm{NO}_{2}$ measurements can also be seen in the figures in comparison to the year 2020. The improvement achieved is visible, again, both in the position of the scatter points as well as the histogram distribution.

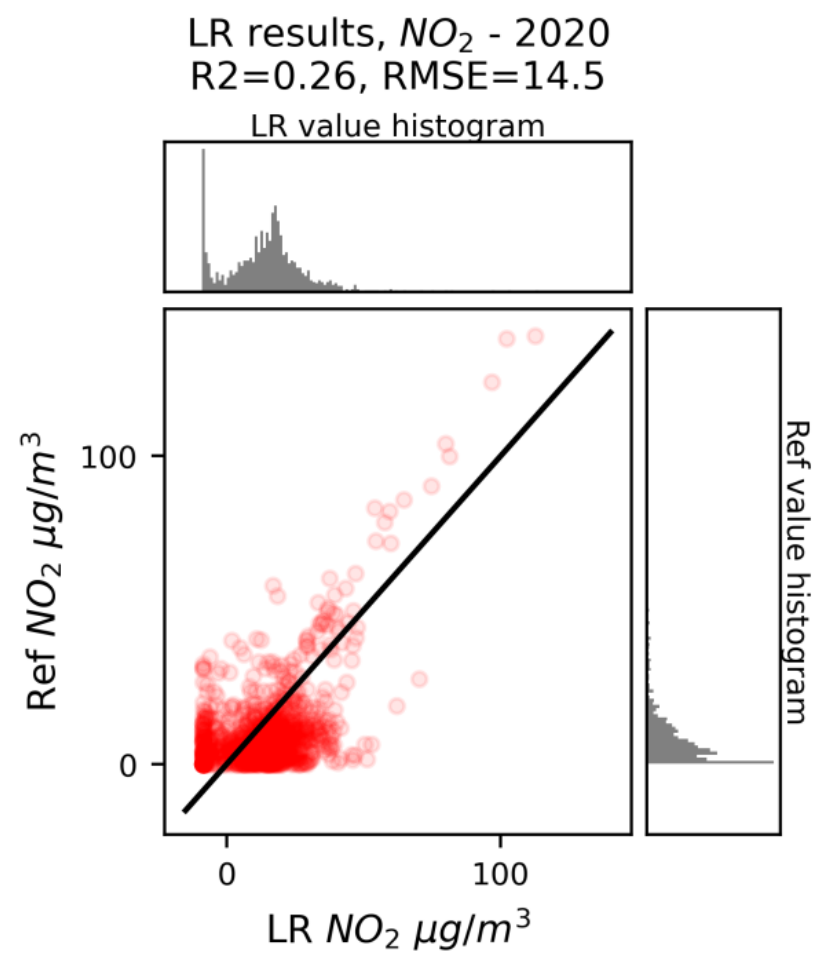

Figure 2. The results of the LR performed in the year 2020. 


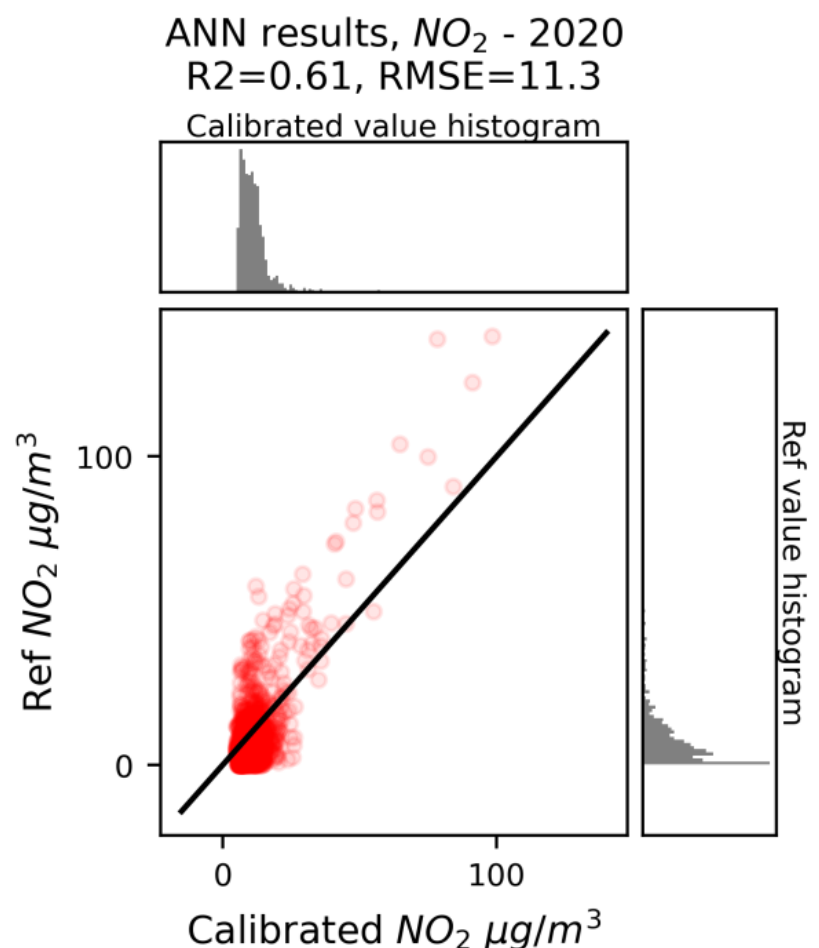

Figure 3. The results of the ANN calibration, trained on data from 2019 and applied to data from 2020.

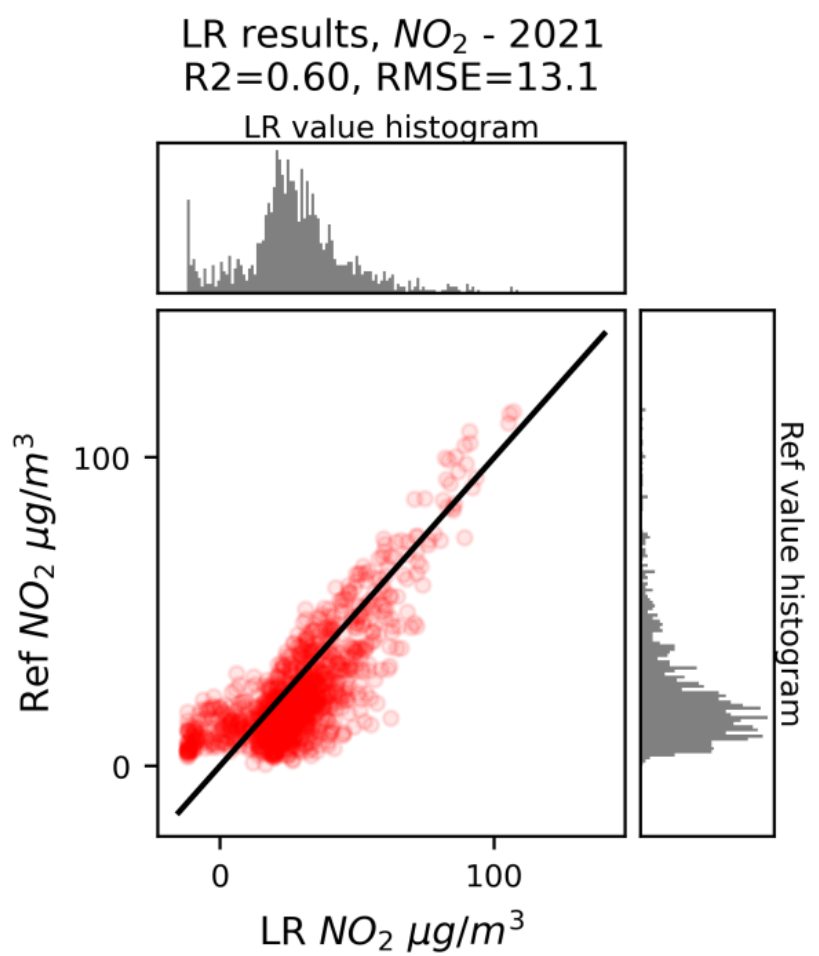

Figure 4. The results of the LR performed in the year 2021. 


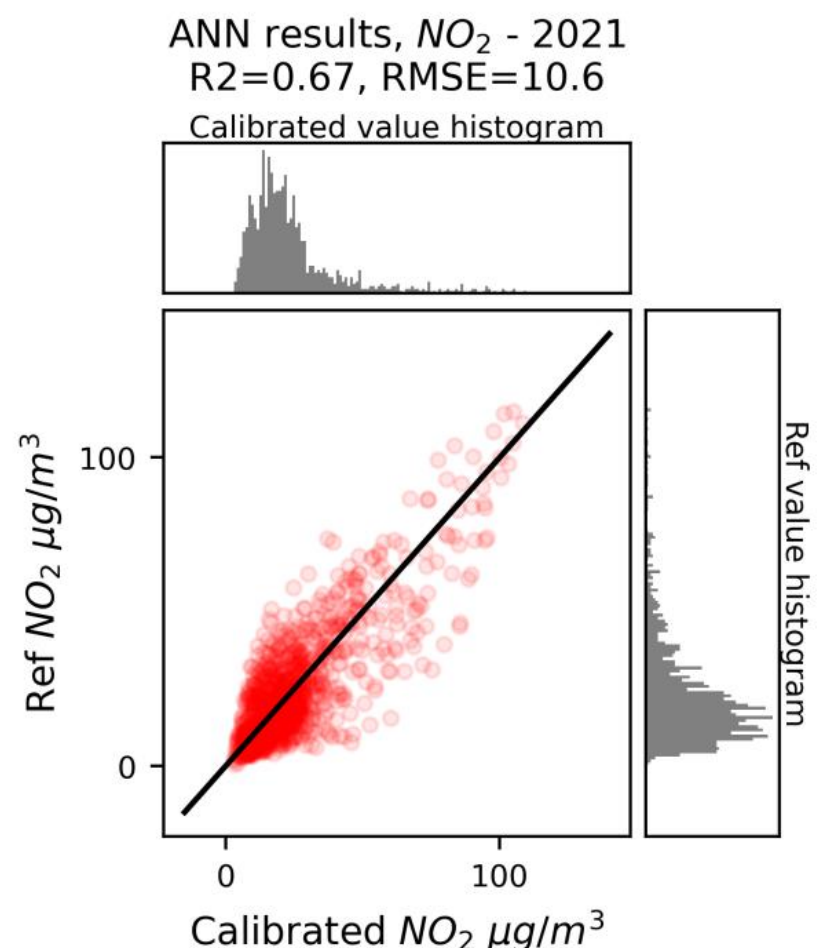

Figure 5. The results of the ANN calibration, trained on data from 2019 as well as 2020 and applied to data from 2021.

When it comes to the $\mathrm{PM}_{10}$ measurements, no significant change in the concentrations throughout the years can be observed in the reference measurements. The low-cost measurements are successfully corrected for 2020 using the ANN, but for 2021 this is not the case. In contrast to the $\mathrm{NO}_{2}$ measurements, the results for 2021 are better when the ANN is trained solely on data from 2020. This can be expected considering the nature of the low-cost $\mathrm{PM}_{10}$ sensors and their degradation. The low-cost $\mathrm{PM}_{10}$ sensors are optical, and they measure the light that is reflected off the particles that enter the sensor chamber. As time passes, the particles accumulate, blocking the entrance to the sensor chamber, resulting in lower $\mathrm{PM}_{10}$ measurements as the particles cannot properly circulate through the sensor chamber and therefore refract less light. Taking this into account, the low-cost sensors' behavior in 2019 is vastly different from their behavior in 2021. Because of this, the ANN gains no benefit from 2019 data as they do not represent the same dependency between the variables that can be observed in 2021. Finally, the improvement for 2021 (when the ANN is trained on 2020 data) is present only for the R2 factor, while the RMSE is higher than the one obtained after LR. This is most likely because of the changes in the baseline of the low-cost sensor, caused by sensor degradation, although the overall behavior of the sensor in 2021 is still similar enough to the one in 2020 to provide an increase in R2.

\section{Conclusions}

The source of $\mathrm{NO}_{2}$ pollution in cities comes from burning fuel (cars, buses, trucks and power plants), so road traffic is the main source of $\mathrm{NO}_{2}$. In accordance with other published work in this area, we have noticed a remarkable decrease in $\mathrm{NO}_{2}$ during the COVID-19 lockdown. On the other hand, pollutants related to domestic heating showed constant or higher levels. $\mathrm{CO}$ and $\mathrm{PM}_{10}$ pollutants in cities are mostly related to individual domestic heating sources (gas, fossil fuels and biomass) and heating plants, thus showing almost the same level for $\mathrm{PM}_{10}$, while $\mathrm{CO}$ showed a slight increase. It has to be noted that the source of PM pollution might not always be local pollution, but could come from remote areas, brought by the wind. It could be observed that the PM concentrations did not decline remarkably despite a reduction in traffic, which leads to the conclusion that 
the PM concentration locally varies according to the dominant PM pollution source in the observed area that corresponds to the specific micro-meteorological climate.

A convolutional ANN has been proposed in this paper to explore the possibilities of modeling the influence of relative humidity and air temperature on the low-cost measurements. We have concluded that for the $\mathrm{CO}$ measurements, no significant improvement could be achieved, but for those of $\mathrm{NO}_{2}$ and $\mathrm{PM}_{10}$ a clear improvement can be observed. When training the developed convolutional network on 2019 data and testing it on 2020 data (during the COVID-19 lockdown), an improvement of 0.346 is achieved for the $\mathrm{NO}_{2}$ $R 2$ value, and an improvement of 0.126 is achieved for the $P_{10} R 2$ value. This shows that the data from a regular period can be used to train a model which can then improve the performance of low-cost sensors even during a different day-to-day regime. It is important to note that our developed model uses sequences of data, and that this choice is made because when the same train/test data splitting methodology was attempted with single measurement points and simpler machine learning models (fully connected ANN and random forest) no improvement could be achieved.

In our future work, we will explore the influence of the lockdown on different pollutants. An exploration of more complex convolutional neural networks will also be performed, including an expansion of the network input parameters (both meteorological and other pollutants) as well as the possibilities of cross-calibration between low-cost sensors.

Author Contributions: Conceptualization, I.V. and D.D.; methodology, D.D and Z.C.; software, I.V.; validation, Z.C.; formal analysis, I.V., Z.C. and D.D.; investigation, I.V. and Z.C.; resources, I.V.; data curation, D.D. and Z.C.; writing — original draft preparation, I.V., Z.C. and D.D.; writing—review and editing, Z.C. and D.D.; visualization, I.V.; supervision, D.D. and Z.C.; project administration, I.V.; funding acquisition, I.V., Z.C. and D.D. All authors have read and agreed to the published version of the manuscript.

Funding: This research was funded by the Ministry of Education, Science and Technological Development of the Republic of Serbia. The APC is partially covered by the Ministry of Education, Science and Technological Development of the Republic of Serbia.

Conflicts of Interest: The authors declare no conflict of interest.

\section{References}

1. The Government of the Republic of Serbia Web Portal, COVID-19. 15 March 2020. Available online: https:/ /www.srbija.gov.rs/ vest/en/151398/state-of-emergency-declared-throughout-serbia.php (accessed on 27 October 2021).

2. UN (United Nations): Air Quality Improvements from COVID Lockdowns Confirmed. Available online: https://news.un.org/ en/story/2021/09/1099092 (accessed on 27 October 2021).

3. Davidović, M.; Dmitrašinović, S.; Jovanović, M.; Radonić, J.; Jovašević-Stojanović, M. Diurnal, Temporal and Spatial Varia-tions of Main Air Pollutants Before and during Emergency Lockdown in the City of Novi Sad (Serbia). Appl. Sci. 2021, 11, 1212. [CrossRef]

4. Donzelli, G.; Cioni, L.; Cancellieri, M.; Morales, A.L.; Suárez-Varela, M.M. The Effect of the COVID-19 Lockdown on Air Quality in Three Italian Medium-Sized Cities. Atmosphere 2020, 11, 1118. [CrossRef]

5. Bar, S.; Parida, B.R.; Mandal, S.P.; Pandey, A.C.; Kumar, N.; Mishra, B. Impacts of partial to complete COVID-19 lockdown on NO2 and PM2.5 levels in major urban cities of Europe and USA. Cities 2021, 117, 103308. [CrossRef] [PubMed]

6. INERIS, M.; Schulz, M.; Mortie, A.; Tsyro, S.; Benedictow, A.; Fagerli, H.; Tarrason, L.; Hamar, P.; Colette, A.; Raux, B.; et al. COVID Impact on Air Quality in Europe A Preliminary Regional Model Analysis. Available online: https:/ / policy.atmosphere. copernicus.eu/reports/CAMS71_COVID_20200626_v1.3.pdf (accessed on 27 September 2021).

7. Mashayekhi, R.; Pavlovic, R.; Racine, J.; Moran, M.D.; Manseau, P.M.; Duhamel, A.; Katal, A.; Miville, J.; Niemi, D.; Peng, S.J.; et al. Isolating the impact of COVID-19 lockdown measures on urban air quality in Canada. Air Qual. Atmos. Health 2021, 14, 1549-1570. [CrossRef]

8. Xiang, J.; Austin, E.; Gould, T.; Larson, T.; Shirai, J.; Liu, Y.; Marshall, J.; Seto, E. Impacts of the COVID-19 responses on traffic-related air pollution in a Northwestern US city. Sci. Total Environ. 2020, 747, 141325. [CrossRef]

9. Motlagh, N.H.; Lagerspetz, E.; Nurmi, P.; Li, X.; Varjonen, S.; Mineraud, J.; Siekkinen, M.; Rebeiro-Hargrave, A.; Hussein, T.; Petaja, T.; et al. Toward Massive Scale Air Quality Monitoring. IEEE Commun. Mag. 2020, 58, 54-59. [CrossRef] 
10. Yu, H.; Li, Q.; Geng, Y.-A.; Zhang, Y.; Wei, Z. AirNet: A Calibration Model for Low-Cost Air Monitoring Sensors Using Dual Sequence Encoder Networks. In Proceedings of the AAAI Conference on Artificial Intelligence, New York, NY, USA, 7-12 February 2020; Association for the Advancement of Artificial Intelligence (AAAI): Menlo Park, CA, USA, 2020; Volume 34, pp. 1129-1136. [CrossRef]

11. Gao, Y.; Dong, W.; Guo, K.; Liu, X.; Chen, Y.; Liu, X.; Bu, J.; Chen, C. Mosaic: A low-cost mobile sensing system for urban air quality monitoring. In Proceedings of the IEEE INFOCOM 2016-The 35th Annual IEEE International Conference on Computer Communications, San Francisco, CA, USA, 10-14 April 2016; pp. 1-9. [CrossRef]

12. Topalovic, D.B.; Davidovic, M.D.; Jovanović, M.; Bartonova, A.; Ristovski, Z.; Jovašević-Stojanović, M. In search of an optimal in-field calibration method of low-cost gas sensors for ambient air pollutants: Comparison of linear, multilinear and artificial neural network approaches. Atmos. Environ. 2019, 213, 640-658. [CrossRef]

13. Casey, J.G.; Hannigan, M.P. Testing the performance of field calibration techniques for low-cost gas sensors in new de-ployment locations: Across a county line and across Colorado. Atmos. Meas. Tech. 2018, 11, 6351-6378. [CrossRef]

14. Casey, J.G.; Collier-Oxandale, A.; Hannigan, M. Performance of artificial neural networks and linear models to quantify 4 trace gas species in an oil and gas production region with low-cost sensors. Sens. Actuators B Chem. 2019, 283, 504-514. [CrossRef]

15. Air Monitoring-ekoNET. Available online: https:/ / ekonet.solutions/air-monitoring/ (accessed on 27 October 2021).

16. B-Series-Alphasense. Available online: https://www.alphasense.com/products/b-series/ (accessed on 27 October 2021).

17. PMS 7003-PM2.5-Plantower Technology. Available online: http://www.plantower.com/en/content/?110.html (accessed on 27 October 2021).

18. How to Evaluate Low-Cost Sensors by Collocation with Federal Reference Method Monitors. Available online: https:/ /www.epa. gov/sites/default/files/2018-01/documents/collocation_instruction_guide.pdf (accessed on 27 October 2021).

19. Teh, H.Y.; Kempa-Liehr, A.W.; Wang, K.I.-K. Sensor data quality: A systematic review. J. Big Data 2020, 7, 1-49. [CrossRef] 